\title{
Kybernetika
}

\section{Gang Li}

On a special class of left-continuous uninorms

Kybernetika, Vol. 54 (2018), No. 3, 427-442

Persistent URL: http://dml.cz/dmlcz/147430

\section{Terms of use:}

(C) Institute of Information Theory and Automation AS CR, 2018

Institute of Mathematics of the Czech Academy of Sciences provides access to digitized documents strictly for personal use. Each copy of any part of this document must contain these Terms of use.

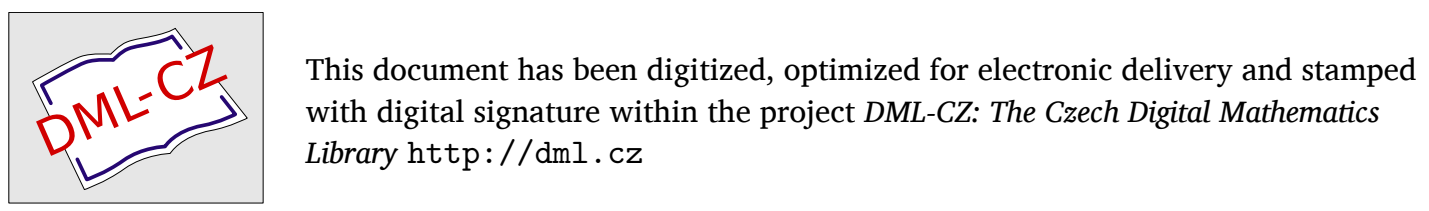




\title{
ON A SPECIAL CLASS OF LEFT-CONTINUOUS UNINORMS
}

\author{
GANG Li
}

This paper is devoted to the study of a class of left-continuous uninorms locally internal in the region $A(e)$ and the residual implications derived from them. It is shown that such uninorm can be represented as an ordinal sum of semigroups in the sense of Clifford. Moreover, the explicit expressions for the residual implication derived from this special class of uninorms are given. A set of axioms is presented that characterizes those binary functions $I:[0,1]^{2} \rightarrow[0,1]$ for which a uninorm $U$ of this special class exists in such a way that $I$ is the residual implications derived from $U$.

Keywords: uninorm, internal operator, ordinal sum, residual implication, triangular subnorm

Classification: $06 \mathrm{~F} 05,03 \mathrm{E} 72,03 \mathrm{~B} 52$

\section{INTRODUCTION}

Triangular norms (t-norms) were originally introduced within the framework of probabilistic metric spaces. Triangular norms and related fuzzy implications also play the important role in the semantics of the fuzzy logics [17, 22]. For example, the continuous t-norms play the role of the logical conjunction in the semantics of BL (basic logic) and left-continuous t-norms in the semantics of MTL (monoidal t-norm based logic). It is well known that the continuous t-norm can be characterized via the ordinal sum construction of Clifford [10. However, a full characterization of left-continuous t-norms is still not known. Many different classes of left-continuous t-norms are constructed in [21, 35. Axiomatic characterization for the residual implications derived from leftcontinuous t-norms have appeared along the time (see the monograph [3]).

Uninorms were introduced by Yager and Rybalov [45] as a generalization of t-norms and t-conorms. Uninorms allows for a neutral element lying anywhere in the unit interval rather than at one or zero as in the case of t-norms and t-conorms. Since the introduction in 1996, uninorms have been studied by many authors from both theoretical and application points of view. The first deep study by Fodor et al. [18] revealed the structure of uninorms. Uninorms and the residual implications derived from them have been successfully used in many application fields where t-norms or t-conorms apply, for example, expert systems [6, 47], decision making [32, 46], fuzzy integrals [23] and fuzzy morphology [8]. On the other hand, the theoretical study of uninorms has been even

DOI: $10.14736 /$ kyb-2018-3-0427 
more extensive [1, 5, 17, 11, 19, 20, 25, 26, 32, 36, 38, 40, 43. It is well known that there exists no continuous proper uninorms. Hence, it is essential and important to study the class of left-continuous uninorms and the residual implications derived from them. In [1], Aguiló et al. have given characterizations for the class of residual implications derived from left-continuous conjunctive uninorms in a similar way as it done for leftcontinuous t-norms. Moreover, a characterization for the particular cases when uninorm $U$ is representable, when $U$ is continuous in $] 0,1\left[^{2}\right.$ and when $U$ is idempotent has also been included in [1].

In this paper, we focus on a special class of left-continuous uninorms locally internal in $A(e)$ (see Definition 2.4). In section 2, we recall some basic results about uninorms and the residual implications derived from them. In section 3 , some properties of this class of uninorms are presented and it is shown that the uninorms can be represented as an ordinal sum of semigroups in the sense of Clifford (see Theorem 3.5). In section 4, we concentrate on the residual implications derived from uninorms and give the explicit expressions for the residual implications derived from this class of uninorms (see Theorem 4.1). Moreover, a set of axioms is presented that characterizes those binary functions $I:[0,1]^{2} \rightarrow[0,1]$ for which a uninorm $U$ of this class exists in such a way that $I$ is the residual implications derived from $U$ (see Theorem 4.2). Hence, the results presented here can be viewed as the complements to those appeared in [1].

\section{PRELIMINARIES}

In this section we will give some basic facts about triangular norms, triangular conorms and uninorms. More details can be found in [2, 22,

Definition 2.1. (Alsina et al. [2], Klement et al. [22]) A triangular subnorm ( $t$ subnorm for short) is a commutative, associative, increasing function $M:[0,1]^{2} \rightarrow[0,1]$ such that $M(x, y) \leq \min (x, y)$ for all $(x, y) \in[0,1]^{2}$. Moreover, if 1 is the neutral element of the t-subnorm, i. e., $M(1, x)=x$ for all $x \in[0,1]$ then $M$ is called triangular norm (t-norm for short). The t-norm often is denoted by $T$. It is obvious that for t-norm $T$, $T(x, y) \leq \min (x, y)$ for all $(x, y) \in[0,1]^{2}$.

A dual operation to a t-subnorm is t-superconorm.

Definition 2.2. (Alsina et al. [2, Klement et al. [22]) A triangular superconorm ( $t$ superconorm for short) is a commutative, associative, increasing function $R:[0,1]^{2} \rightarrow$ $[0,1]$ such that $R(x, y) \geq \max (x, y)$ for all $(x, y) \in[0,1]^{2}$. Moreover, if 0 is the neutral element of the t-superconorm, i. e., $R(0, x)=x$ for all $x \in[0,1]$ then $R$ is called triangular conorm (t-conorm for short). The t-conorm often is denoted by $S$. It is obvious that for t-conorm $S, S(x, y) \geq \max (x, y)$ for all $(x, y) \in[0,1]^{2}$.

Definition 2.3. (Yager and Rybalov [45]) A uninorm is a commutative, associative, increasing function $U:[0,1]^{2} \rightarrow[0,1]$ such that $U(e, x)=x$ for all $x \in[0,1]$, where $e \in[0,1]$ is often called the neutral element. If $U\left(x_{0}, x_{0}\right)=x_{0}$ for some $x_{0} \in[0,1]$ then $x_{0}$ is called the idempotent element of $U$.

We summarize some fundamental results from [18]. 
It is clear that the function $U$ becomes a t-norm when $e=1$ and a t-conorm when $e=0$. A uninorms can be also taken as a bipolar t-conorm on $[-1,1]$ (see [32]). For any uninorm we have $U(0,1) \in\{0,1\}$. A uninorm $U$ such that $U(0,1)=0$ is called conjunctive and if $U(0,1)=1$ then it is called disjunctive.

Throughout this paper, we exclusively consider uninorms with a neutral element $e$ strictly between 0 and 1 .

With any uninorm $U$ with neutral element $e \in] 0,1$, we can associate two binary operations $T_{U}, S_{U}:[0,1]^{2} \rightarrow[0,1]$ defined by

$$
T_{U}(x, y)=\frac{1}{e} \cdot U(e x, e y)
$$

and

$$
S_{U}(x, y)=\frac{1}{1-e}(U(e+(1-e) x, e+(1-e) y)-e)
$$

It is easy to see that $T_{U}$ is a t-norm and that $S_{U}$ is a t-conorm. In other words, on $[0, e]^{2}$ any uninorm $U$ is determined by a t-norm $T_{U}$, and on $[e, 1]^{2}$ any uninorm $U$ is determined by a t-conorm $S_{U} ; T_{U}$ is called the underlying t-norm, and $S_{U}$ is called the underlying t-conorm. Hence, for uninorm $U$, we have $U(x, y) \leq \min (x, y)$ for all $(x, y) \in[0, e]^{2}$ and $U(x, y) \geq \max (x, y)$ for all $(x, y) \in[e, 1]^{2}$. Let us denote the remaining part of the unit square by $A(e)$, i. e.,

$$
A(e)=[0,1]^{2} \backslash\left([0, e]^{2} \cup[e, 1]^{2}\right) .
$$

On the set $A(e)$, any uninorm $U$ is bounded by the minimum and the maximum of its arguments, i. e., for any $(x, y) \in A(e)$ it holds that

$$
\min (x, y) \leq U(x, y) \leq \max (x, y) .
$$

Note that uninorm (t-norm, t-subnorm, t-conorm) can be defined on any interval $I \subseteq[0,1]$ by an isomorphic transformation from $I$ to $[0,1]$.

The classes of $\mathcal{U}_{\min }$ (respectively $\mathcal{U}_{\max }$ ) and representable uninorms were introduced in [18. After this, some other classes of uninorms were discussed like idempotent uninorms in [7, 29, 42], uninorms continuous in the open square $] 0,1\left[^{2}\right.$ in [14, 20] and uninorms with continuous underlying operators [15, 19, 24, 26, 33, 34]. Moreover, the relations between different classes of uninorms were also discussed in [16, 27, 31.

There exists another class of uninorms [11, 16, 28, which has been extensively studied in the literature, i. e., those uninorms that are locally internal in a region of $[0,1]^{2}$.

Definition 2.4. (Drygas et al. [16]) A uninorm $U$ with neutral element $e \in] 0,1[$ is called locally internal in a region $R \subseteq[0,1]^{2}$ if it satisfies $U(x, y) \in\{x, y\}$ for all $(x, y) \in R$.

The class of uninorms locally internal on the boundary of $[0,1]^{2}$ has been discussed in [28] and the authors have shown that such uninorms can be represented as an ordinal sum of semigroups in the sense of Clifford [10].

Note that the term locally internal (also called conservative in [37]) is first used to describe the fact that the values of a monotonic and associative binary function 12 at 
all point $(x, y) \in[0,1]^{2}$ must equal to one of its argument. A full characterization of such binary functions was given using a decreasing function $g$ dividing the region where the function takes the value of the minimum and the maximum. These results were used later to characterize the idempotent uninorms [7, 29, 42] and also for the discussion of those uninorms which are locally internal in $A(e)$. Some results about the latter class of uninorms are listed here.

Definition 2.5. (Baets et al. 99]) A decreasing function $g:[0,1] \rightarrow[0,1]$ is called $I d$-symmetrical if its completed graph $F_{g}$ satisfies

$$
(x, y) \in F(g) \Leftrightarrow(y, x) \in F(g),
$$

where

$$
\begin{aligned}
& F_{g}=\{(x, g(x)): x \in[0,1]\} \cup\{(0, y): y>g(0)\} \cup\{(1, y): y<g(1)\} \\
& \cup\left\{(s, y): s^{-} \leq y \leq s^{+}\right\},
\end{aligned}
$$

$s$ is any discontinuity point of $g$ and $s^{-}, s^{+}$are the corresponding lateral limits, i.e., $s^{-}=\lim _{x \rightarrow s-} g(x), s^{+}=\lim _{x \rightarrow s+} g(x)$,

Some properties about the $I d$-symmetrical function was given in [13].

Proposition 2.6. (Drygas [13]) Let $g:[0,1] \rightarrow[0,1]$ be a decreasing function with fixed point $e \in] 0,1[$. Then $g$ is $I d$-symmetrical if and only if $g$ satisfies the following conditions:

(i) $\inf \{y: g(y)=g(x)\} \leq g(g(x)) \leq \sup \{y: g(y)=g(x)\}$ for all $x \in[0,1]$,

(ii) $g$ is constant, say $g(x)=s$ in the interval $] p, q[$ with $p<q$, where

$$
p=\inf \{x \in[0,1]: g(x)=s\}, q=\sup \{x \in[0,1]: g(x)=s\},
$$

if and only if, $s \in] 0,1$ [ is a discontinuity point of $g$ or $s \in\{0,1\}$ and it is satisfied that

$$
p= \begin{cases}\lim _{x \rightarrow s+} g(x) & s<1 \\ 0 & s=1\end{cases}
$$

and

$$
q= \begin{cases}\lim _{x \rightarrow s-} g(x) & s>0 \\ 1 & s=0\end{cases}
$$

Remark 2.7. Note that if $g$ is strictly decreasing and continuous on an interval $] a, b[\subseteq$ $[0,1]$ then $g(] a, b[)=] c, d[\subseteq[0,1], g$ is also strictly decreasing and continuous on $] c, d[$ and $g(g(x))=x$ for all $x \in] a, b[\cup] c, d[$.

Theorem 2.8. (Drygas [13, Ruiz-Aguilera et al. [42]) Let $U$ be a uninorm with neutral element $e \in] 0,1$ [ and locally internal in $A(e)$. Then there exists a decreasing function $g:[0,1] \rightarrow[0,1]$ with $g(e)=e$ which is $I d$-symmetrical, in such a way that $U$ is given in the region $A(e)$ by

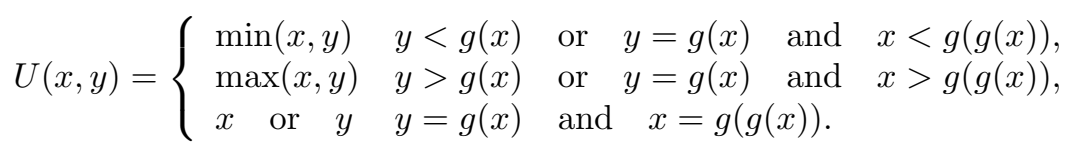


The function $g$ in Theorem 2.8 is often called the associated function of the uninorm $U$.

Unfortunately, a full characterization for the class of uninorms in Theorem 2.8 is still open. An interesting problems arise:

Problem: Whether the class of uninorms locally internal in region $A(e)$ can also be represented as an ordinal sum of semigroups in the sense of Clifford?

In this paper, we focus on the conjunctive, left-continuous uninorms which are locally internal in the region $A(e)$ and the residual implications derived from them which both play the important role in fuzzy logics [4], fuzzy relational equations [4] and fuzzy morphology [8]. Note that the residual implications derived from some classes of uninorms have been discussed in [1, 39, 41, respectively.

Now, we recall here some facts on implications.

Definition 2.9. (Baczyński and Jayaram [3]) A binary function $I:[0,1]^{2} \rightarrow[0,1]$ is said to be an implication function, or an implication, if it satisfies:

(I1) $I(x, z) \geq I(y, z)$ when $x \leq y$ for all $z \in[0,1]$.

(I2) $I(x, y) \leq I(x, z)$ when $y \leq z$ for all $x \in[0,1]$.

(I3) $I(0,0)=I(1,1)=1, I(1,0)=0$.

Definition 2.10. (Baczyński and Jayaram [3]) Let $U$ be a uninorm. The residual operation derived from $U$ is the binary operation given by

$$
I_{U}(x, y)=\sup \{z \in[0,1]: U(x, z) \leq y\}
$$

for all $x, y \in[0,1]$.

For the residual operation derived from $U$, the following result holds.

Proposition 2.11. (De Baets and Fodor [5]) Let $U$ be a uninorm. Then the residual operation $I_{U}$ is implication if and only if the following condition holds:

$$
U(x, 0)=0 \text { for all } x<1 .
$$

The implication $I_{U}$ in Proposition 2.11 is usually called $R U$-implication.

Note that all conjunctive uninorms but also many disjunctive ones satisfy the condition (5). However, when we focus on left-continuous uninorms, we immediately have that uninorm $U$ satisfies condition (5) if and only if $U$ is conjunctive.

As for t-norm, the left-continuity of uninorm is an important property since the corresponding $R U$-implication $I_{U}$ satisfies the residuation property.

Proposition 2.12. (Aguiló et al. [1) Let $U$ be a conjunctive uninorm and $I_{U}$ its residual implication. Then $U$ is left-continuous if and only if $I_{U}$ satisfies the residuation property:

$$
U(x, y) \leq z \Leftrightarrow I_{U}(x, z) \geq y \text { for all } x, y \in[0,1]
$$


Moreover, when $U$ is left-continuous, some important properties are satisfied.

Proposition 2.13. (Aguiló et al. 1]) Let $U$ be a left-continuous conjunctive uninorm with neutral element $e \in] 0,1\left[\right.$. Then $I_{U}$ satisfies

(i) Counterpart for uninorms of the ordering property:

$$
I_{U}(x, y) \geq e \Leftrightarrow x \leq y, \quad x, y \in[0,1] .
$$

(ii) Exchange principle:

$$
I_{U}\left(x, I_{U}(y, z)\right)=I_{U}\left(y, I_{U}(x, z)\right), \quad x, y, z \in[0,1] .
$$

An axiomatic characterization of the residual implications derived from conjunctive left-continuous uninorm $U$ was given in [1]. Moreover, a characterization for the particular cases when $U$ is representable, when $U$ is continuous in $] 0,1\left[^{2}\right.$ and when $U$ is idempotent were also given in [1].

Theorem 2.14. (Aguiló et al. [1]) Let $I:[0,1]^{2} \rightarrow[0,1]$ be a function and $\left.e \in\right] 0,1[$. The following statements are equivalent:

(i) $I$ is an $R U$-implication derived from a left-continuous uninorm $U$ with neutral element $e$.

(ii) $I$ satisfies $(I 2),\left(O P_{U}\right),(E P)$ and $I(x, \cdot)$ is right-continuous for all $x \in[0,1]$.

Moreover, in this case the uninorm $U$ must be conjunctive and it is given by:

$$
U(x, y)=\inf \{z \in[0,1]: I(x, z) \geq y\} .
$$

\section{THE STRUCTURE OF THE SPECIAL CLASS OF UNINORMS}

In this section, we focus on the class of conjunctive, left-continuous uninorms locally internal in the region $A(e)$, that is, those uninorms that satisfy that $U(x, y) \in\{x, y\}$ for all $(x, y) \in A(e)$. Note that the general uninorms locally internal in the region $A(e)$ have been studied in [13, 15].

By Theorem 2.8, the following result holds firstly.

Proposition 3.1. Let $U$ be a conjunctive, left-continuous uninorm with neutral element $e \in] 0,1[$ and locally internal in $A(e)$. Then there exists a decreasing, left-continuous function $g:[0,1] \rightarrow[0,1]$ with $g(0)=1, g(e)=e$ which is $I d$-symmetrical, in such a way that $U$ is given in the region $A(e)$ by

$$
U(x, y)= \begin{cases}\min (x, y) & y \leq g(x) \\ \max (x, y) & y>g(x)\end{cases}
$$

Proof. By Theorem 2.8 there exists a unary left-continuous function $g$ satisfies the conditions in Proposition 3.1 and Eq. (7) holds since the left-continuity of $U$. Since $U$ is conjunctive, we have $U(0,1)=0, g(0)=1$. 
Proposition 3.2. Let $U$ be a conjunctive, left-continuous uninorm with neutral element $e \in] 0,1$ [ such that $U$ is locally internal in $A(e)$ and let $g$ be its associated function. If $g$ is strictly decreasing and continuous on $] a, b[$, then

(i) $g(x)$ is an idempotent element of $U$ for all $x \in] a, b[$, and

(ii) $x$ is an idempotent element of $U$ for all $x \in] a, b]$.

Proof. (i) The proof is equal to the proof of Proposition 5 in [16].

(ii) By the similar proof of Proposition 5 in [16], we can prove that $y \in] a, b[$ are idempotent elements of $U$. By the left-continuity of $U$, we obtain that $b$ is idempotent element too.

Proposition 3.3. Let $U$ be a conjunctive, left-continuous uninorm with neutral element $e \in] 0,1$ [ such that $U$ is locally internal in $A(e)$ and let $g$ be its associated function. If $g$ is constant, say $g(x)=s$ in the interval $] a, b]$ with $a=\inf \{x \in[0,1]: g(x)=s\}<b=$ $\sup \{x \in[0,1]: g(x)=s\}$, then the following statements hold.

(i) If $] a, b] \subseteq[0, e]$ then $s$ is an idempotent element of $U$ and $U(x, y)=\min (x, y)$ for all $(x, y) \in[0, a] \times] a, e] \cup] a, e] \times[0, a]$.

(ii) If $] a, b] \subseteq[e, 1]$ then $a$ is an idempotent elements of $U$ and $U(x, y)=\max (x, y)$ for all $(x, y) \in[e, a] \times[a, b] \cup[a, b] \times[e, a]$.

Proof. Note that the case $e \in] a, b[$ can not appear since $g$ is $I d$-symmetrical.

(i) By the similar proof of Proposition 6 in [16, we can prove that $s$ is an idempotent element of $U$. Now, we consider the point $a$ :

- If there exists $l<a$ such that $g$ is strictly decreasing and continuous on $] l, a[$. Then by Proposition 3.2, the point $a$ is an idempotent element of $U$. Due to the fact that $e$ is the neutral element of $U$ and the monotonicity and the commutativity of $U$, we have $U(x, y)=\min (x, y)$ for all $(x, y) \in[0, a] \times] a, e] \cup] a, e] \times[0, a]$.

- If $a$ is only a discontinuity point of the function $g$ then we can prove that $U(u, v)=$ $u$ for all $u, v$ such that $u<a<v<b$ by the equal proof of Proposition 6 in 16]. By the left-continuity of $U$, we have $U(a, v)=a$ for all $a<v<b$ and $U(u, b)=u$ for all $u \leq a$. Due to the fact that $e$ is the neutral element of $U$ and the monotonicity and the commutativity of $U$, we have $U(x, y)=\min (x, y)$ for all $(x, y) \in[0, a] \times] a, e] \cup] a, e] \times[0, a]$.

(ii) Suppose $] a, b] \subseteq[e, 1]$. Then $s \leq e$. Considering the point $a$, we have the following two cases:

- If there exists $l<a$ such that $g$ is strictly decreasing and continuous on $] l, a[$. Then by Proposition 3.2 , the point $a$ is an idempotent element of $U$. 
- If $a$ is only a discontinuity point of the function $g$ then we denote the left limit $\lim _{x \rightarrow a-} g(x)$ of $g$ at point $a$ by $l$. So, $l \leq e$. Taking $\left.y \in\right] s, l[$ and $u, v$ such that $e \leq u<a<v<b$, we have $g(u) \geq l>y>s=g(v)$ and $U(u, v) \geq \max (u, v)=$ $v$. So, $g(U(u, v)) \leq g(v)<y$. By Proposition 3.1. the commutativity and the associativity of $U$, we have

$$
\begin{aligned}
U(u, v) & =\max (U(u, v), y) \\
& =U(U(u, v), y) \\
& =U(v, U(u, y)) \\
& =U(v, \min (u, y)) \\
& =U(v, y) \\
& =\max (v, y) \\
& =v
\end{aligned}
$$

It means that $U(u, v)=v$ for all $u, v$ such that $e \leq u<a<v<b$. By the left-continuity of $U$, we have $U(a, v)=v$ for all $a<v \leq b$. So, $U(a, a) \leq a$ by the monotonicity of $U$. Since $U(a, a) \geq a$, we have $U(a, a)=a$.

Due to the fact that $e$ is the neutral element of $U$ and the monotonicity and the commutativity of $U$, we have $U(x, y)=\max (x, y)$ for all $(x, y) \in[e, a] \times[a, b] \cup[a, b] \times[e, a]$.

The new structure of the class of conjunctive, left-continuous uninorms which are locally internal in $A(e)$ is presented here. Firstly, we need the following results of Clifford introduced in 10 .

Theorem 3.4. Let $A \neq \emptyset$ be a totally ordered set and $\left(G_{\alpha}\right)_{\alpha \in A}$ with $G_{\alpha}=\left(X_{\alpha}, *_{\alpha}\right)$ be a family of semigroups. Assume that for all $\alpha, \beta \in A$ with $\alpha<\beta$ the sets $X_{\alpha}$ and $X_{\beta}$ are either disjoint or that $X_{\alpha} \cap X_{\beta}=\left\{x_{\alpha \beta}\right\}$, where $x_{\alpha \beta}$ is both the neutral element of $G_{\alpha}$ and the annihilator of $G_{\beta}$ and where for each $\gamma \in A$ with $\alpha<\gamma<\beta$ we have $X_{\gamma}=\left\{x_{\alpha \beta}\right\}$. Put $X=\bigcup_{\alpha \in A} X_{\alpha}$ and define the binary operation $*$ on $X$ by

$$
x * y= \begin{cases}x *_{\alpha} y & \text { if }(x, y) \in X_{\alpha} \times X_{\alpha}, \\ x & \text { if }(x, y) \in X_{\alpha} \times X_{\beta} \text { and } \quad \alpha<\beta, \\ y & \text { if }(x, y) \in X_{\alpha} \times X_{\beta} \text { and } \beta<\alpha .\end{cases}
$$

Then $G=(X, *)$ is a semigroup. The semigroup $G$ is commutative if and only if for each $\alpha \in A$ the semigroup $G_{\alpha}$ is commutative.

According to three different cases of the associated function $g$ at point 0 , the structures of uninorms are given respectively in the following theorem.

Theorem 3.5. Let $U$ be a conjunctive, left-continuous uninorm with neutral element $e \in] 0,1$ [ such that $U$ is locally internal in $A(e)$ and let $g$ be its associated function. Then one of the following statements holds.

(i) If there exists the largest interval $[0, r$ [ such that $g$ is strictly decreasing and continuous on $[0, r[$, then the semigroup $G=([0,1], U)$ is an ordinal sum of the 
semigroups $\left.\left.G_{1}=(([0, r] \cup] g(r), 1]\right), U\right)$ and $G_{2}=([r, g(r)], U)$. Moreover, in this case, the restriction of $U$ to $([0, r] \cup] g(r), 1])^{2}$ is a conjunctive, left-continuous uninorms defined on $[0, r] \cup] g(r), 1]$ with the neutral element $r$, and the restriction of $U$ to $[r, g(r)]^{2}$ is a conjunctive, left-continuous uninorms defined on $[r, g(r)]$ with the neutral element $e$.

(ii) If there exists the largest interval $[0, r]$ such that $g$ is constant on $[0, r]$, i. e., $g(x)=1$ for all $x \in[0, r]$. Then the semigroup $G=([0,1], U)$ is an ordinal sum of the semigroups $G_{1}=\left(([0, r], U)\right.$ and $\left.\left.G_{2}=(] r, 1\right], U\right)$. Moreover, in this case, the restriction of $U$ to $[0, r]^{2}$ is a left-continuous t-subnorm defined on $[0, r]$, and the restriction of $U$ to $] r, 1]^{2}$ is a left-continuous uninorms defined on $\left.] r, 1\right]$ with the neutral element $e$.

(iii) If $g$ is discontinuous at point 0 , then the semigroup $G=([0,1], U)$ is an ordinal sum of the semigroups $G_{1}=([0, s], U)$ and $\left.\left.G_{2}=(] s, 1\right], U\right)$, where $s=\lim _{x \rightarrow 0+} g(x)$. Moreover, in this case, the restriction of $U$ to $[0, s]^{2}$ is a conjunctive, left-continuous uninorm defined on $[0, s]$ with the neutral element $e$, and the restriction of $U$ to ]$s, 1]^{2}$ is a left-continuous t-superconorm defined on $\left.] s, 1\right]$.

Proof. (i) By Proposition [2.6 and Remark 2.7 $g(] 0, r[)=] g(r), 1]$ and $g$ is strictly decreasing and continuous on $] g(r), 1$. By Proposition 3.2 and the trivial fact that 0,1 are idempotent elements of $U$, arbitrary $x \in[0, r] \cup] g(r), 1]$ is an idempotent element of $U$. Note that $g(r) \in[e, 1[$. Then $U(g(r), g(r))=g(r)$. In fact, since $U(y, y)=y$ for all $y \geq g(r), U(g(r), g(r)) \leq g(r)$. Moreover, due to the fact that $U(g(r), g(r)) \geq$ $\max (g(r), g(r))=g(r)$, it holds $U(g(r), g(r))=g(r)$. So, $g(r)$ is an idempotent element of $U$.

Due to Proposition 3.1, the monotonicity of $U$ and the fact that $e$ is the neutral element of $U, U(x, y)=\min (x, y)$ for all $(x, y) \in[0, r] \times[r, e] \cup[r, e] \times[0, r]$ and $U(x, y)=$ $\max (x, y)$ for all $(x, y) \in[g(r), 1] \times[e, g(r)[\cup[e, g(r)] \times[g(r), 1]$. Moreover, $U(x, y) \in\{x, y\}$ for every $(x, y) \in[0, r] \times] g(r), 1] \cup] g(r), 1] \times[0, r]$ by Proposition 3.1. Thus, $[0, r] \cup] g(r), 1]$ is closed under $U$. Since $U(r, y)=y$ for all $y \in[0, r] \cup] g(r), 1]$ by Proposition 3.1 the restriction of $U$ to $[0, r] \cup] g(r), 1])^{2}$ is a conjunctive, left-continuous uninorms defined on $([0, r] \cup] g(r), 1]$ with the neutral element $r$.

By the monotonicity of $U$ and $U(r, r)=r, U(g(r), g(r))=g(r)$, we have $U(x, y) \geq r$ for all $(x, y) \in[r, e]^{2}$ and $U(x, y) \leq g(r)$ for every $(x, y) \in[e, g(r)]^{2}$. Due to Proposition 3.1. $U(x, y)=\max (x, y)$ for all $(x, y) \in] g(r), 1] \times[r, g(r)] \cup[r, g(r)] \times] g(r), 1]$ and $U(x, y)=$ $\min (x, y)$ for all $(x, y) \in[0, r] \times[e, g(r)] \cup[e, g(r)] \times[0, r]$. Hence, $[r, g(r)]$ is closed under $U$ and the restriction of $U$ to $[r, g(r)]^{2}$ is a conjunctive, left-continuous uninorms defined on $[r, g(r)])$ with the neutral element $e$. So, $U(r, y)=U(y, r)=\min (r, y)=r$ for all $y \in[r, g(r)]$.

Now, we construct two semigroups $\left.\left.G_{1}=(([0, r] \cup] g(r), 1]\right), U\right), G_{2}=([r, g(r)], U)$ and a totally ordered set $A=\{1,2\}$ equipped with the natural order of the real numbers. It is obvious that $U$ is an ordinal sum in the sense of Clifford of the semigroups $G 1, G 2$.

(ii) By Proposition 3.1, Proposition 2.6 and Proposition 3.3, $U(x, y)=\min (x, y)$ for every $(x, y) \in[0, r] \times] r, 1] \cup] r, 1] \times[0, r]$. So, $[0, r]$ is closed under $U$ and the restriction of $U$ to $[0, r]^{2}$ is a left-continuous t-subnorm defined on $[0, r]$. Now, we prove that there 
exists no $\left.\left(x_{0}, y_{0}\right) \in\right] r, e\left[^{2}\right.$ such that $U\left(x_{0}, y_{0}\right)=r$. On the contrary, suppose that there exists $\left.\left(x_{0}, y_{0}\right) \in\right] r, e\left[{ }^{2}\right.$ such that $U\left(x_{0}, y_{0}\right)=r$. Then $U\left(U\left(x_{0}, y_{0}\right), 1\right)=U(r, 1)=r$ and $U\left(x_{0}, U\left(y_{0}, 1\right)\right)=U\left(x_{0}, \max \left(y_{0}, 1\right)\right)=U\left(x_{0}, 1\right)=\max \left(x_{0}, 1\right)=1$, a contradiction with the associativity of $U$. Hence, $] r, 1]$ is closed under $U$ and the restriction of $U$ to $] r, 1]^{2}$ is a left-continuous uninorms defined on $] r, 1]$ with the neutral element $e$. We construct two semigroups $\left.G_{1}=\left(([0, r], U), G_{2}=(] r, 1\right], U\right)$ and a totally ordered set $A=\{1,2\}$ equipped with the natural order of the real numbers. It is obvious that $U$ is an ordinal sum in the sense of Clifford of the semigroups $G 1, G 2$.

(iii) For the discontinuity point 0 of $g$, let us denote by $s$ the corresponding right limit $s=\lim _{x \rightarrow 0+} g(x)$. By Proposition [2.6, $g$ is constant in the interval ] $\left.s, 1\right]$. From Proposition 3.3, $s$ is an idempotent element of $U$. Moreover, by Proposition 3.2, $U(x, y)=\max (x, y)$ for all $(x, y) \in[e, s] \times[s, 1] \cup[s, 1] \times[e, s]$. By the monotonicity of $g$, we know that $g(x) \geq s$ for all $x \in[0, e]$. So, by Proposition 3.1 $U(x, y)=\max (x, y)$ for all $(x, y) \in] 0, e] \times] s, 1] \cup] s, 1] \times] 0, e]$. It is obvious that $[0, s]$ is closed under $U$ and the restriction of $U$ to $[0, s]^{2}$ is a conjunctive, left-continuous uninorm defined on $[0, s]$ with the neutral element $e$. Moreover, $U(x, y) \geq \max (x, y)$ for all $(x, y) \in] s, 1]^{2}$. Hence, ] $\left.s, 1\right]$ is closed under $U$ and the restriction of $U$ to $] s, 1]^{2}$ is a left-continuous t-superconorm defined on $] s, 1]$. We construct two semigroups $\left.G_{1}=\left(([0, s], U), G_{2}=(] s, 1\right], U\right)$ and a totally ordered set $A=\{1,2\}$ equipped with the natural order of the real numbers. It is obvious that $U$ is an ordinal sum in the sense of Clifford of the semigroups $G 1, G 2$.

Remark 3.6. Note that Theorem 3.5 partially offers a positive answer to the Problem in Section 2.

\section{THE RESIDUAL IMPLICATION DERIVED FROM UNINORMS}

In this section, we focus on the residual implication derived from the conjunctive, leftcontinuous uninorm locally internal in the region $A(e)$.

Theorem 4.1. Let $U$ be a conjunctive, left-continuous uninorm with neutral element $e \in] 0,1$ [ such that $U$ is locally internal in $A(e)$ and let $g$ be its associated function. Then the residual implication $I_{U}$ derived from $U$ is given by

$$
I_{U}(x, y)= \begin{cases}\max (g(x), y) & x \leq y \leq e \text { or } x \leq e \leq y \\ \min (g(x), y) & e \leq y<x \text { or } y \leq e \leq x \\ e \cdot I_{T_{U}}\left(\frac{x}{e}, \frac{y}{e}\right) & y<x \leq e \\ e+(1-e) \cdot I_{S_{U}}\left(\frac{x-e}{1-e}, \frac{y-e}{1-e}\right) & e \leq x \leq y\end{cases}
$$

Pr o of. We divide the proof in some cases.

- $x \leq y \leq e$. Then $y \leq e \leq g(x)$. In this case, we have $U(x, y) \leq \min (x, y)=x$. If we take $z$ satisfying $e \leq z \leq g(x)$, then $U(x, z)=\min (x, z)=x \leq y$. Moreover, if we take $z$ satisfying $g(x)<z, U(x, z)=\max (x, z)=z>y$, and then

$$
I_{U}(x, y)=\sup \{z: z \in[0,1], U(x, z) \leq y\}=g(x)=\max (g(x), y) .
$$


- $x \leq e \leq y, y \leq g(x)$. In this case, we have $U(x, y)=\min (x, y)=x \leq y$. If we take $z$ satisfying $y \leq z \leq g(x)$, then $U(x, z)=\min (x, z)=x \leq y$. Moreover, if we take $z$ satisfying $g(x)<z, U(x, z)=\max (x, z)=z>y$, and then

$$
I_{U}(x, y)=\sup \{z: z \in[0,1], U(x, z) \leq y\}=g(x)=\max (g(x), y) .
$$

- $x \leq e \leq y, g(x)<y$. In this case, we have $U(x, y)=\max (x, y)=y$. If we take $z$ satisfying $g(x)<z \leq y$, then $U(x, z)=\max (x, z)=z \leq y$. Moreover, if we take $z$ satisfying $y<z, U(x, z)=\max (x, z)=z>y$, and then

$$
I_{U}(x, y)=\sup \{z: z \in[0,1], U(x, z) \leq y\}=y=\max (g(x), y) .
$$

- $e \leq y<x$. Then $g(x) \leq e \leq y$. In this case, we have $U(x, y) \geq \max (x, y)=x$. If we take $z$ satisfying $z \leq g(x)$, then $U(x, z)=\min (x, z)=z \leq y$. Moreover, if we take $z$ satisfying $g(x)<z, U(x, z)=\max (x, z)>y$, and then

$$
I_{U}(x, y)=\sup \{z: z \in[0,1], U(x, z) \leq y\}=g(x)=\min (g(x), y) .
$$

- $y \leq e \leq x, y \leq g(x)$. In this case, we have $U(x, y)=\min (x, y)=y$. If we take $z$ satisfying $x \geq z>y$, then $U(x, z) \geq \min (x, z)=z>y$ and

$$
I_{U}(x, y)=\sup \{z: z \in[0,1], U(x, z) \leq y\}=y=\min (g(x), y) .
$$

- $y \leq e \leq x, g(x)<y$. In this case, we have $g(x) \leq e$ and $U(x, y)=\max (x, y)=$ $x \geq y$. If $x=y=e$ then $I_{U}(x, y)=I_{U}(e, e)=e=g(e)=\min (g(x), y)$. In the sequent, without loss of generality, we assume that $y<x$. If we take $z$ satisfying $z \leq g(x)$, then $U(x, z)=\min (x, z)=z \leq y$. Moreover, if we take $z$ satisfying $g(x)<z, U(x, z)=\max (x, z)>y$, and then

$$
I_{U}(x, y)=\sup \{z: z \in[0,1], U(x, z) \leq y\}=g(x)=\min (g(x), y) .
$$

- $y<x \leq e$. In this case, we have $U(x, y) \leq \min (x, y)=y$. Due to the fact that $U(x, z) \geq \min (x, z)=x>y$ for all $z \in] e, 1]$, we have

$$
\begin{aligned}
I_{U}(x, y) & =\sup \{z: z \in[0,1], U(x, z) \leq y\} \\
& =\sup \left\{z: z \in[0, e], e \cdot T_{U}\left(\frac{x}{e}, \frac{z}{e}\right) \leq y\right\} \\
& =e \cdot \sup \left\{\frac{z}{e}: \frac{z}{e} \in[0,1], T_{U}\left(\frac{x}{e}, \frac{z}{e}\right) \leq \frac{y}{e}\right\} \\
& =e \cdot I_{T_{U}}\left(\frac{x}{e}, \frac{y}{e}\right)
\end{aligned}
$$

- $e \leq x \leq y$. In this case, we have $U(x, y) \geq \max (x, y)=y$. Due to the fact that $U(x, z) \leq \max (x, z)=x \leq y$ for all $z \in[0, e]$, we have

$$
\begin{aligned}
I_{U}(x, y) & =\sup \{z: z \in[0,1], U(x, z) \leq y\} \\
& =\sup \left\{z: z \in[e, 1], e+(1-e) \cdot S_{U}\left(\frac{x-e}{1-e}, \frac{z-e}{1-e}\right) \leq y\right\} \\
& =e+(1-e) \cdot \sup \left\{\frac{z-e}{1-e}: \frac{z-e}{1-e} \in[0,1], S_{U}\left(\frac{x-e}{1-e}, \frac{z-e}{1-e}\right) \leq \frac{y-e}{1-e}\right\} \\
& =e+(1-e) \cdot I_{S_{U}}\left(\frac{x-e}{1-e}, \frac{y-e}{1-e}\right) .
\end{aligned}
$$


From Theorem 4.1, we can derive the following result.

Theorem 4.2. Let $I:[0,1]^{2} \rightarrow[0,1]$ be a function and $\left.e \in\right] 0,1[$. The following statements are equivalent:

(i) $I$ is an $R U$-implication derived from a left-continuous uninorm $U$ with neutral element $e$ which is locally internal in $A(e)$.

(ii) $I$ satisfies $(I 2),\left(O P_{U}\right),(E P), I(x, \cdot)$ is right-continuous for all $x \in[0,1]$, and there exists a function $g:[0, e] \rightarrow[e, 1]$ such that $I(x, y)=\max (g(x), y)$ for all $x \leq e \leq y$ or $x \leq y \leq e$.

Moreover, in this case the uninorm $U$ must be conjunctive and it is given by:

$$
U(x, y)=\inf \{z \in[0,1]: I(x, z) \geq y\} .
$$

P r o of. If $U$ is a left-continuous uninorm locally internal in $A(e)$, then $I$ clearly satisfied all conditions in (ii) by Theorem 2.14 and Theorem 4.1 .

Conversely, if $I$ satisfies all conditions in (ii) then by Theorem $2.14 I$ must be the residual implication derived from the conjunctive, left-continuous uninorm given by

$$
U(x, y)=\inf \{z \in[0,1]: I(x, z) \geq y\}
$$

Thus, we only need to prove that in our case such uninorm must be locally internal in $A(e)$.

Since for all $x \in[0, e], x=U(x, e)=\inf \{z \in[0,1]: I(x, z) \geq e\}=\inf \{z \in[x, e]:$ $I(x, z) \geq e\}=\inf \{z \in[x, e]: \max (g(x), z) \geq e\}$, we have $g(x) \geq e$ for all $x \in[0, e]$.

Since $g(e)=U(e, g(e))=\inf \{z \in[0,1]: I(e, z) \geq g(e)\}=\inf \{z \in[e, 1]: I(e, z) \geq$ $g(e)\}=\inf \{z \in[e, 1]: \max (g(e), z) \geq g(e)\}=e$, we have $g(e)=e$.

For arbitrary $x_{1} \leq x_{2} \leq e$, we have $I\left(x_{1}, e\right)=\max \left(g\left(x_{1}\right), e\right)=g\left(x_{1}\right)$ and $I\left(x_{2}, e\right)=$ $\max \left(g\left(x_{2}\right), e\right)=g\left(x_{2}\right)$. Since $I$ is an implication and is decreasing with respect to the first argument, $g\left(x_{1}\right) \geq g\left(x_{2}\right)$, i. e., $g$ is decreasing.

Since $0=U(0,1)=\inf \{z \in[0,1]: I(0, z) \geq 1\}=\inf \{z \in[0, e]: I(0, z) \geq 1\}=$ $\inf \{z \in[0, e]: \max (g(0), z) \geq 1\}=\inf \{z \in[0, e]: g(0) \geq 1\}$, we have $g(0)=1$.

For all $x \leq e \leq y$, we have $x \leq U(x, y) \leq y$ by Eq. (1).

- If $y>g(x)$ then

$$
\begin{aligned}
U(x, y) & =\inf \{z \in[0,1]: I(x, z) \geq y\} \\
& =\inf \{z \in[x, 1]: I(x, z) \geq y\} \\
& =\inf \{z \in[x, 1]: \max (g(x), z) \geq y\} \\
& =\inf \{z \in[x, 1]: z \geq y\} \\
& =y
\end{aligned}
$$

Hence, $U(x, y)=\max (x, y)=y$. 
- If $y \leq g(x)$ then

$$
\begin{aligned}
U(x, y) & =\inf \{z \in[0,1]: I(x, z) \geq y\} \\
& =\inf \{z \in[x, 1]: I(x, z) \geq y\} \\
& =\inf \{z \in[x, 1]: \max (g(x), z) \geq y\} \\
& =\inf \{z \in[x, 1]\} \\
& =x
\end{aligned}
$$

Hence, $U(x, y)=\min (x, y)=x$.

So, the uninorm $U$ is locally internal in $A(e)$ by the commutativity of $U$.

\section{CONCLUSIONS}

One of the most used classes of conjunctions and fuzzy implications is the class of leftcontinuous t-norms and their residual implications, but recently, many other kinds of functions are used in this framework. In this paper, we discuss the structure of the conjunctive, left-continuous uninorms locally internal in $A(e)$ and give an axiomatic characterization of those binary functions $I:[0,1]^{2} \rightarrow[0,1]$ that are the residual implications derived from this special class of uninorms, in a similar way to the characterization of residual implications derived from left-continuous uninorms. Our results can be taken as complements to those in [1].

Note that using the so-called weak law of importation $(W L I)$, another characterization of residual implication derived from this special class of uninorms can be given by Theorem 31 in [30. We say that a function $I:[0,1]^{2} \rightarrow[0,1]$ satisfies the weak law of importation if there exist a conjunctive, commutative and nondecreasing function $F:[0,1]^{2} \rightarrow[0,1]$ such that $I(F(x, y), z)=I(x, I(y, z))$ for all $x, y, z \in[0,1]$ and then we say that $I$ satisfies $(W L I)$ with the function $F$.

Theorem 5.1. Let $I:[0,1]^{2} \rightarrow[0,1]$ be a function and $\left.e \in\right] 0,1[$. The following statements are equivalent:

(i) $I$ is an $R U$-implication derived from a left-continuous uninorm $U$ which is locally internal in $A(e)$.

(ii) $I$ satisfies $\left(O P_{U}\right)$ and $(W L I)$ with a conjunctive, left-continuous uninorm $U$.

\section{ACKNOWLEDGEMENTS}

The author was supported by the National Natural Science Foundation of China (Grant No. $61403220,61573211)$. 
[1] I. Aguiló, J. Suñer, and J. Torrens:

A characterization of residual implications derived from left-continuous uninorms. Inform. Sci. 180 (2010), 3992-4005. DOI:10.1016/j.ins.2010.06.023

[2] C. Alsina, M. J. Frank, and B. Schweizer: Associative Functions. Triangular Norms and Copulas. World Scientific, New Jersey 2006. DOI:10.1142/9789812774200

[3] M. Baczyński and B. Jayaram: Fuzzy Implications. Springer, Berlin, Herdelberg 2008.

[4] B. De Baets: An order-theoretic approach to solving sup-T equations. In: Fuzzy Set Theory and Advanced Mathemtical Applications (D. Ruan, ed.), Kluwer, Dordrecht 1995, pp. 67-87. DOI:10.1007/978-1-4615-2357-4_3

[5] B. De Baets and J. Fodor: Residual operators of uninorms. Soft Comput. 3 (1999), 89-100. DOI:10.1007/s005000050057

[6] B. De Baets and J. Fodor: Van Melle's combining function in MYCIN is a representable uninorm: An alternative proof. Fuzzy Sets Systems 104 (1999), 133-136. DOI:10.1016/s0165-0114(98)00265-6

[7] B. De Baets: Idempotent uninorms. Eur. J. Oper. Res. 118 (1998), 631-642. DOI:10.1016/s0377-2217(98)00325-7

[8] B. De Baets, N. Kwasnikowska, and E. Kerre: Fuzzy morphology based on uninorms. In: Seventh IFSA World Congress, Prague, 220 (1997), 215-220.

[9] B. De Baets, J. Fodor, D. Ruiz-Aguilera, and J. Torrens: Idempotent uninorms on finite ordinal scales. Int. J. Uncertain. Fuzziness Knowl.-Based Syst. 17 (2009), 1-14. DOI:10.1142/s021848850900570x

[10] A.H. Clifford: Naturally totally ordered commutative semigroups. Amer. J. Math. 76 (1954), 631-646. DOI:10.2307/2372706

[11] O. Csiszár and J. Fodor: On uninorms with fixed values along their border. Ann. Univ. Sci. Bundapest., Sect. Com. 42 (2014), 93-108.

[12] E. Czogała and J. Drewniak: Associative monotonic operations in fuzzy set theory. Fuzzy Sets Systems 12 (1984), 249-269. DOI:10.1016/0165-0114(84)90072-1

[13] P. Drygaś: Discussion of the structure of uninorms. Kybernetika 41 (2005), 213-226. DOI:10.1016/j.fss.2015.05.018

[14] P. Drygaś: On the structure of continuous uninorms. Kybernetika 43 (2007), 183-196.

[15] P. Drygaś: On properties of uninorms with underlying t-norm and t-conorm given as ordinal sums. Fuzzy Sets Systems 161 (2010), 149-157. DOI:10.1016/j.fss.2009.09.017

[16] P. Drygaś, D. Ruiz-Aguilera, and J. Torrens: A characterization of a class of uninorms with continuous underlying operators. Fuzzy Sets Systems 287 (2016), 137-153. DOI:10.1016/j.fss.2015.07.015

[17] F. Esteva and L. Godo: Monoidal t-norm based logic: owards a logic for left-continuous t-norms. Fuzzy Sets Systems 124 (2001), 271-288. DOI:10.1016/s0165-0114(01)00098-7

[18] J. Fodor, R. R. Yager, and A. Rybalov: Structure of uninorms. Int. J. Uncertain. Fuzziness Knowl.-Based Syst. 5 (1997), 411-427. DOI:10.1142/s0218488597000312

[19] J. Fodor and B. De Baets: A single-point characterization of representable uninorms. Fuzzy Sets Systems 202 (2012), 89-99. DOI:10.1016/j.fss.2011.12.001 
[20] S. Hu and Z. Li: The structure of continuous uninorms. Fuzzy Sets Systems 124 (2001), 43-52. DOI:10.1016/s0165-0114(00)00044-0

[21] S. Jenei: A note on the ordinal sum theorem and its consequence for the construction of triangular norms. Fuzzy Sets Systems 126 (2002), 199-205. DOI:10.1016/s01650114(01)00040-9

[22] E. P. Klement, R. Mesiar, and E. Pap: Triangular Norms. Kluwer Academic Publishers, Dordrecht 2000.

[23] E. P. Klement, R. Mesiar, and E. Pap: Integration with respect to decomposable measures, based on a conditionally distributive semiring on the unit interval. Int. J. Uncertain. Fuzziness Knowl.-Based Syst. 8 (2000), 707-717. DOI:10.1142/s0218488500000514

[24] G. Li, H-W. Liu, and J. Fodor: Single-point characterization of uninorms with nilpotent underlying t-norm and t-conorm. Int. J. Uncertain. Fuzziness Knowl.-Based Syst. 22 (2014), 591-604. DOI:10.1142/s0218488514500299

[25] G. Li, H-W. Liu, and J. Fodor: On almost equitable uninorms. Kybernetika 51(4) (2015), 699-711. DOI:10.14736/kyb-2015-4-0699

[26] G. Li and H-W. Liu: Distributivity and conditional distributivity of a uninorm with continuous underlying operators over a continuous t-conorm. Fuzzy Sets Systems 287 (2016), 154-171. DOI:10.1016/j.fss.2015.01.019

[27] G. Li and H-W. Liu: On Relations Between Several Classes of Uninorms. In: Fan TH., Chen SL., Wang SM., Li YM. (eds) Quantitative Logic and Soft Computing 2016. Advances in Intelligent Systems and Computing, vol. 510. Springer, 2017, pp. 251-259. DOI:10.1007/978-3-319-46206-6_25

[28] G. Li and H-W. Liu: On properties of uninorms locally internal on the boundary. Fuzzy Sets Systems 332 (2017), 116-128. DOI:10.1016/j.fss.2017.07.014

[29] J. Martin, G. Mayor, and J. Torrens: On locally internal monotonic operations. Fuzzy Sets Systems 137(1) (2003), 27-42. DOI:10.1016/s0165-0114(02)00430-x

[30] S. Massanet and J. Torrens: The law of implication versus the exchange principle on fuzzy implications. Fuzzy Sets Systems 168 (2011), 47-69. DOI:10.1016/j.fss.2010.12.012

[31] M. Mas, S. Massanet, D. Ruiz-Aguilera, and J. Torrens: A survey on the existing classes of uninorms. J. Intell. Fuzzy Systems 29(3) (2015), 1021-1037. DOI:10.3233/ifs-151728

[32] A. Mesiarová: Multi-polar t-conorms and uninorms. Inform. Sci. 301 (2015), 227-240. DOI:10.1016/j.ins.2014.12.060

[33] A. Mesiarová: Characterization of uninorms with continuous underlying t-norm and tconorm by their set of discontinuity points. IEEE Trans. Fuzzy Systems PP (2017), in press.

[34] A. Mesiarová: Characterization of uninorms with continuous underlying t-norm and tconorm by means of the ordinal sum construction. Int. J. Approx. Reason. 87 (2017), 176-192. DOI:10.1016/j.ijar.2017.01.007

[35] C. Noguera, F. Esteva, and L. Godo: Generalized continuous and left-continuous t-norms arising from algebraic semantics for fuzzy logics. Inform. Sci. 180 (2010), 1354-1372. DOI:10.1016/j.ins.2009.12.011

[36] M. Petrík and R. Mesiar: On the structure of special classes of uninorms. Fuzzy Sets Systems 240 (2014), 22-38. DOI:10.1016/j.fss.2013.09.013

[37] M. Pouzet, I. G. Rosenberg, and M. G. Stone: A projection property. Algebra Univers. 36(2) (1996), 159-184. DOI:10.1007/bf01234102 
[38] F. Qin and B. Zhao: The distributive equations for idempotent uninorms and nullnorms. Fuzzy Sets Systems 155 (2005), 446-458. DOI:10.1016/j.fss.2005.04.010

[39] D. Ruiz and J. Torrens: Residual implications and co-implications from idempotent uninorms. Kybernetika 40 (2004), 21-38.

[40] D. Ruiz and J. Torrens: Distributivity and conditional distributivity of a uninorm and a continuous t-conorm. IEEE Trans. Fuzzy Systems 14 (2006), 2, 180-190. DOI:10.1109/tfuzz.2005.864087

[41] D. Ruiz-Aguilera and J. Torrens: R-implications and S-implications from uninorms continuous in $] 0,1\left[^{2}\right.$ and their distributivity over uninorms. Fuzzy Sets Systems 160 (2009), 832-852. DOI:10.1016/j.fss.2008.05.015

[42] D. Ruiz-Aguilera, J. Torrens, B. De Baets, J. Fodor: Some remarks on the characterization of idempotent uninorms. In: IPMU 2010, LNAI 6178, Eds. E.Hüllermeier, R.Kruse and F.Hoffmann, Springer-Verlag Berlin Heidelberg 2010, pp. 425-434. DOI:10.1007/978-3-64214049-5_44

[43] D. Ruiz-Aguilera and J. Torrens: A characterization of discrete uninorms having smooth underlying operators. Fuzzy Sets Syst. 268 (2015), 44-58. DOI:10.1016/j.fss.2014.10.020

[44] M. Takács: Uninorm-based models for FLC systems. J. Intell. Fuzzy Systems 19 (2008), 65-73.

[45] R. Yager and A. Rybalov: Uninorm aggregation operators. Fuzzy Sets Systems 80 (1996), 111-120. DOI:10.1016/0165-0114(95)00133-6

[46] R. Yager and A. Rybalov: Bipolar aggregation using the uninorms. Fuzzy Optim. Decis. Making 10 (2011), 59-70. DOI:10.1007/s10700-010-9096-8

[47] R. Yager: Uninorms in fuzzy systems modeling. Fuzzy Sets Systems 122 (2001), 167-175. DOI:10.1016/s0165-0114(00)00027-0

Gang Li, Faculty of Science, Qilu University of Technology, Ji'nan, Shandong 250353. P. R. China.

e-mail: sduligang@163.com 